\title{
ON AN EXPLICIT ZERO-FREE REGION FOR THE DEDEKIND ZETA-FUNCTION
}

ETHAN S. LEE

\begin{abstract}
We establish new explicit zero-free regions for the Dedekind zetafunction. Two key elements of our proof are a non-negative, even, trigonometric polynomial and explicit upper bounds for the explicit formula of the so-called differenced logarithmic derivative of the Dedekind zeta-function. The improvements we establish over the last result of this kind come from two sources. First, our computations use a polynomial which has been optimised by simulated annealing for a similar problem. Second, we establish sharper upper bounds for the aforementioned explicit formula.
\end{abstract}

\section{INTRODUCTION}

Let $K$ be an algebraic number field and $L$ be a normal extension of $K$ with Galois group $G=\operatorname{Gal}(L / K)$. Suppose $d_{L}, d_{K}$ denote the absolute values of the respective discriminant, $n_{L}=[L: \mathbb{Q}]$ and $n_{K}=[K: \mathbb{Q}]$. The Dedekind zeta-function of $L$ is denoted and defined for $\mathfrak{R e}(s)>1$ by

$$
\zeta_{L}(s)=\sum_{\mathfrak{P}} \frac{1}{N(\mathfrak{P})^{s}}
$$

where $\mathfrak{P}$ ranges over the non-zero ideals of $\mathcal{O}_{L}$. If $n_{L}=a+b$, then one can also consider the completed zeta-function

$$
\begin{aligned}
& \xi_{L}(s)=s(s-1) d_{L}^{\frac{s}{2}} \gamma_{L}(s) \zeta_{L}(s) \text { such that } \\
& \gamma_{L}(s)=\pi^{-\frac{a s}{2}} \Gamma\left(\frac{s}{2}\right)^{a} \pi^{-\frac{b(s+1)}{2}} \Gamma\left(\frac{s+1}{2}\right)^{b} .
\end{aligned}
$$

Here, $\xi_{L}$ is an entire function satisfying the functional equation $\xi_{L}(s)=\xi_{L}(1-s)$. It can be seen that $\zeta_{L}$ is meromorphic on the complex plane with exactly one simple pole at $s=1$. Let $\mathcal{P}$ denote a prime ideal of $K$ and $P$ denote a prime ideal of $L$. If $\mathcal{P}$ is unramified in $L$, then the Artin symbol,

$$
\left[\frac{L / K}{\mathcal{P}}\right]
$$

denotes the conjugacy class of Frobenius automorphisms corresponding to prime ideals $P \mid \mathcal{P}$. For each conjugacy class $C \subset G$, the prime ideal counting function is

$$
\pi_{C}(x, L / K)=\#\left\{\mathcal{P}: \mathcal{P} \text { unramified in } L,\left[\frac{L / K}{\mathcal{P}}\right]=C, N_{K}(\mathcal{P}) \leq x\right\} .
$$


In 1926, Chebotarëv [2] proved the Chebotarëv density theorem, which states that

$$
\pi_{C}(x, L / K) \sim \frac{\# C}{\# G} \operatorname{Li}(x)=\frac{\# C}{\# G} \int_{2}^{x} \frac{d t}{\log t} \text { as } x \rightarrow \infty .
$$

For example, if $L=K=\mathbb{Q}$, then the Chebotarëv density theorem restates the prime number theorem. Moreover, if $\omega_{\ell}=e^{\frac{2 \pi i}{\ell}}$ is the $\ell$ th root of unity, $K=\mathbb{Q}$ and $L=\mathbb{Q}\left(\omega_{\ell}\right)$, then the Chebotarëv density theorem identifies with the Dirichlet theorem for primes in arithmetic progressions.

In 1977, Lagarias-Odlyzko 9] provided explicit estimates for the error term of the Chebotarëv density theorem. There are two results contained therein; one version assumes the generalised Riemann hypothesis (GRH) for $\zeta_{L}$ and the other does not. Their error term is effectively computable, dependent only on $x, n_{L}, d_{L}$ and $\frac{\# C}{\# G}$.

Under the GRH for $\zeta_{L}$, one can obtain the best possible effective results. Without assuming the GRH for $\zeta_{L}$, the better the zero-free region for $\zeta_{L}$ one has, the better the effective result one can achieve. Therefore, the objective of this paper is to improve the best known, explicit zero-free region for $\zeta_{L}$, given by Kadiri [6] in 2012. We recall two famous forms of zero-free regions for the Riemann zeta-function.

Classical zero-free region. In 1899, de la Vallée Poussin [3] famously proved that there exists a positive constant $R$ such that $\zeta$ is non-zero in the region $s=\sigma+i t$ such that $t \geq T$ and

$$
\sigma \geq 1-\frac{1}{R \log t}
$$

The best known zero-free region for $\zeta$ of this kind is attributed to MossinghoffTrudgian [12, who verified (11) for $R \approx 5.573$ and $T=2$.

Koborov-Vinogradov zero-free region. In 1958, Koborov 8] and Vinogradov [15] independently demonstrated that there exists a positive constant $R_{1}$ such that $\zeta$ is non-zero in the region $s=\sigma+i t$ such that $t \geq T$ and

$$
\sigma \geq 1-\frac{1}{R_{1}(\log t)^{\frac{2}{3}}(\log \log t)^{\frac{1}{3}}} .
$$

The best known zero-free region for $\zeta$ of this kind is attributed to Ford [4, who has verified (2) for $R_{1}=57.54$ and $T=3$. Ford [4] also establishes the zero-free region (2) for large $t$ with $R_{1}=49.13$.

Naturally, the closest form of the zero-free region for $\zeta_{L}$ will also depend on the extra variables $d_{L}$ and $n_{L}$. However, the method we adopt is based on de la Vallée Poussin's method for determining the classical zero-free region for $\zeta$. One complication is that a so-called exceptional zero could exist inside a zero-free region for $\zeta_{L}$. If this exceptional zero exists, then it must be simple and real.

Kadiri [6, Theorem 1.1] was the last to re-purpose de la Vallée Poussin's proof (using Stečkin's [14] so-called differencing trick) to obtain a zero-free region for $\zeta_{L}$. In this paper, we will establish Theorem 1, a new zero-free region for $\zeta_{L}$ which builds upon Kadiri's zero-free region for $\zeta_{L}$. We will also establish Theorem 2, which will reveal more information pertaining to the exceptional zero. 
Theorem 1. Suppose $\left(C_{1}, C_{2}, C_{3}, C_{4}\right)=(12.2411,9.5347,0.05017,2.2692)$, then $\zeta_{L}(\sigma+i t)$ is non-zero for

$$
\sigma \geq 1-\frac{1}{C_{1} \log d_{L}+C_{2} \cdot n_{L} \log |t|+C_{3} \cdot n_{L}+C_{4}} \text { and }|t| \geq 1 .
$$

Theorem 2. For asymptotically large $d_{L}$ and $R=12.43436, \zeta_{L}(\sigma+i t)$ has at most one zero in the region

$$
\sigma \geq 1-\frac{1}{R \log d_{L}} \text { and }|t|<1
$$

If this exceptional zero exists, then it is simple and real.

Kadiri [6] established (3) with $\left(C_{1}, C_{2}, C_{3}, C_{4}\right)=(12.55,9.69,3.03,58.63)$. To yield Theorem 1, we will follow a similar process to Kadiri, but observe some improvements. An important step in the proof of Theorem 1 is to choose a polynomial $p_{n}(\varphi)$ from the so-called the class of non-negative, trigonometric polynomials of degree $n$; denoted and defined by

$$
P_{n}:=\left\{p_{n}(\varphi)=\sum_{k=0}^{n} a_{k} \cos (k \varphi): p_{n}(\varphi) \geq 0 \text { for all } \varphi, a_{k} \geq 0 \text { and } a_{0}<a_{1}\right\} .
$$

Whereas Kadiri worked with polynomials from $P_{4}$, we will use the same polynomial from $P_{16}$ as Mossinghoff-Trudgian [12. This polynomial has been optimised by simulated annealing for computations pertaining to their computations for the zero-free region for $\zeta$. This amendment contributed all of the improvements that can be seen for $C_{1}$ and $C_{2}$. In fact, if one re-runs Kadiri's computations, only updating the polynomial, then this establishes (3i) with $\left(C_{1}, C_{2}, C_{3}, C_{4}\right)=$ $(12.2411,9.5347,3.3492,57.7027)$.

Another improvement follows from improvements we have made to [11, Lemma 2] from McCurley. In particular, we improve explicit values for $\mathcal{S}(k)$, a computable constant dependent on $k \in \mathbb{N}$. These improvements will contribute almost all of the improvement one observes for $C_{3}$.

Kadiri [6] also established (44) with $R=12.7305$. To yield Theorem 2, we will recycle bounds from [6, §3] and apply the same higher degree polynomial from $P_{16}$. A corollary of the method we use to establish Theorem 2 is an improvement to a well-known region by Stark 13. However, because we only update the polynomial for this method, we cannot improve Stark's result further than [ 6 , Corollary 1.2] already does.

Finally, if an exceptional zero $\beta_{1}$ exists, then one can enlarge the zero-free region in Theorem 2 using the Deuring-Heilbronn phenomenon [10. This was one of the key ingredients in work by Ahn-Kwon [1, Zaman [16] and Kadiri-Ng-Wong [7, which pertains to the least prime ideal in the Chebotarëv density theorem.

Remark. The method of proof which we follow does not use Heath-Brown's version of Jensen's formula [5, Lemma 3.2], although this might yield better zero-free regions than those we can obtain using this method. This is partially because there does not exist a general sub-convexity bound for general number fields, so it is 
difficult to apply his approach in the number field setting - see Kadiri [6] for an excellent explanation of this.

Acknowledgements. I would like to thank my supervisor, Tim Trudgian, for bringing this project to my attention, as well as his continued support. I would also like to thank my other colleagues at UNSW Canberra for their support throughout this process and Kevin Ford for correcting a referential error.

\section{Proof of Theorem 1}

The set-up of our proof for Theorem 1 is the same as that which Kadiri uses in her proof of [6, Theorem 1.1], which has a similar shape to Stečkin's argument [14] for $\zeta$. Suppose $t \geq 1$. We introduce some definitions, which will hold for the remainder of this paper:

- $\kappa=\frac{1}{\sqrt{5}}$

- $s_{k}=\sigma+i k t$ such that $k \in \mathbb{N}, 1<\sigma<1+\varepsilon$ for some $0<\varepsilon \leq 0.15$;

- $s_{k}^{\prime}=\sigma_{1}+i k t$ such that $\sigma_{1}=\frac{1+\sqrt{1+4 \sigma^{2}}}{2}$.

Note that $\sigma_{1}$ depends on $\sigma$, so for convenience we will write $\sigma_{1}(a)$ to denote the the value of $\sigma_{1}$ at $\sigma=a$. To prove Theorem 1, we will isolate a non-trivial zero $\rho=\beta+i t$ of $\zeta_{L}$ such that $\beta>1-\varepsilon \geq 0.85$, choose a polynomial $p_{n}(\varphi)$ from $P_{n}$, and consider the function

$$
S(\sigma, t)=\sum_{k=0}^{n} a_{k} f_{L}(\sigma, k t)
$$

such that

$$
\begin{aligned}
f_{L}(\sigma, k t) & =-\mathfrak{R e}\left(\frac{\zeta_{L}^{\prime}}{\zeta_{L}}\left(s_{k}\right)-\kappa \frac{\zeta_{L}^{\prime}}{\zeta_{L}}\left(s_{k}^{\prime}\right)\right) \\
& =\sum_{0 \neq \mathfrak{P} \subset \mathcal{O}_{L}} \Lambda(\mathfrak{P})\left(N(\mathfrak{P})^{-\sigma}-\kappa N(\mathfrak{P})^{-\sigma_{1}}\right) \cos (k t \log (N(\mathfrak{P})) .
\end{aligned}
$$

It follows that

$$
S(\sigma, t)=\sum_{0 \neq \mathfrak{P} \subset \mathcal{O}_{L}} \Lambda(\mathfrak{P})\left(N(\mathfrak{P})^{-\sigma}-\kappa N(\mathfrak{P})^{-\sigma_{1}}\right) p_{n}(t \log (N(\mathfrak{P})) \geq 0 .
$$

On the other hand, we can utilise the explicit formula [9, (8.3)],

$$
-\frac{\zeta_{L}^{\prime}}{\zeta_{L}}\left(s_{k}\right)=\frac{\log d_{L}}{2}+\frac{1}{s_{k}}+\frac{1}{s_{k}-1}+\frac{\gamma_{L}^{\prime}}{\gamma_{L}}\left(s_{k}\right)-\frac{1}{2} \sum_{\varrho \in Z\left(\zeta_{L}\right)}\left(\frac{1}{s_{k}-\varrho}+\frac{1}{s_{k}-\bar{\varrho}}\right) .
$$

Here, $Z\left(\zeta_{L}\right)$ denotes the set of non-trivial zeros of $\zeta_{L}$. One can use (5) to show

$$
0 \leq S(\sigma, t) \leq S_{1}+S_{2}+S_{3}+S_{4},
$$


where $F(s, z)=\mathfrak{R e}\left(\frac{1}{s-z}+\frac{1}{s-1+\bar{z}}\right)$ such that

$$
\begin{aligned}
& S_{1}=-\sum_{k=0}^{n} a_{k} \sum_{\varrho \in Z\left(\zeta_{L}\right)} \mathfrak{R e}\left(\frac{1}{s_{k}-\varrho}-\frac{\kappa}{s_{k}^{\prime}-\varrho}\right), \\
& S_{2}=\frac{1-\kappa}{2}\left(\sum_{k=0}^{n} a_{k}\right) \log d_{L}, \\
& S_{3}=\sum_{k=0}^{n} a_{k}\left(F\left(s_{k}, 1\right)-\kappa F\left(s_{k}^{\prime}, 1\right)\right), \text { and } \\
& S_{4}=\sum_{k=0}^{n} a_{k} \mathfrak{R e}\left(\frac{\gamma_{L}^{\prime}\left(s_{k}\right)}{\gamma_{L}\left(s_{k}\right)}-\kappa \frac{\gamma_{L}^{\prime}\left(s_{k}^{\prime}\right)}{\gamma_{L}\left(s_{k}^{\prime}\right)}\right) .
\end{aligned}
$$

We will choose $n=16$, so that we can apply Mossinghoff-Trudgian's polynomial $p_{16}(\varphi) \in P_{16}$ from 12 . Taking $n=16, S_{2}$ is directly computable, and we find upper bounds for $S_{1}, S_{3}$, and $S_{4}$ in Sections 2.1, 2.2. and 2.3. The resulting upper bound for $S_{1}+S_{2}+S_{3}+S_{4}$ will depend on $\beta, \sigma, t$, the coefficients of $p_{16}(\varphi)$ and $\varepsilon$, therefore we may use (6) and rearrange the inequality to obtain Theorem 1 in Section 2.4

\subsection{Upper bound for $S_{1}$.}

Lemma 3 (Stečkin [14]). Suppose $s=\sigma+i t$ with $1<\sigma \leq 1.25$ and $z \in \mathbb{C}$. If $0<\mathfrak{R e}(z)<1$, then

$$
F(s, z)-\kappa F\left(s_{1}^{\prime}, z\right) \geq 0 .
$$

Moreover, if $\mathfrak{I m}(z)=\mathfrak{I m}(s)=t$ and $\frac{1}{2} \leq \mathfrak{R e}(z)<1$, then

$$
\mathfrak{R e}\left(\frac{1}{s-1+\bar{z}}\right)-\kappa F\left(s_{1}^{\prime}, z\right) \geq 0 \text {. }
$$

Note that $\kappa$ is the largest value such that (7) holds. This subsection is not an improvement on 6. Lemma 2.3], rather a repeat for the purpose of clarity. By the positivity condition (7) in Lemma 3. we have

$$
\ell\left(s_{k}\right):=\sum_{\varrho \in Z\left(\zeta_{L}\right)} \mathfrak{R e}\left(\frac{1}{s_{k}-\varrho}-\frac{\kappa}{s_{k}^{\prime}-\varrho}\right) \leq \kappa F\left(s_{k}^{\prime}, \rho\right)-F\left(s_{k}, \rho\right) .
$$

If $k=1$, then (8) implies that

$$
\ell\left(s_{1}\right) \leq-\frac{1}{\sigma-\beta}-\frac{1}{\sigma-1+\beta}+\frac{\kappa}{\sigma_{1}-\beta}+\frac{\kappa}{\sigma_{1}-1+\beta}=-\frac{1}{\sigma-\beta}+g(\sigma, \beta) .
$$

We see that $g(\sigma, \beta)<g(1,1)$ and $g(1,1)$ is small and negative, so $\ell\left(s_{1}\right) \leq-\frac{1}{\sigma-\beta}$. Moreover, if $k \neq 1$, then (8) implies that $\ell\left(s_{k}\right) \leq 0$ by (7). One can package the preceding observations into the following lemma.

Lemma 4. Isolate a zero $\rho=\beta+i t \in Z\left(\zeta_{L}\right)$ such that $\beta \geq 1-\varepsilon \geq 0.85$, then

$$
\ell(\sigma+i k t) \leq \begin{cases}-\frac{1}{\sigma-\beta} & \text { if } k=1 \\ 0 & \text { if } k \neq 1\end{cases}
$$

Therefore, $S_{1} \leq-\frac{a_{1}}{\sigma-\beta}$. 
2.2. Upper bound for $S_{3}$. Suppose that

$$
\begin{aligned}
\Sigma_{k}(\sigma, t) & :=F(\sigma+i k t, 1)-\kappa F\left(\sigma_{1}+i k t, 1\right) \\
& =\frac{\sigma}{\sigma^{2}+k^{2} t^{2}}+\frac{\sigma-1}{(\sigma-1)^{2}+k^{2} t^{2}}-\kappa \frac{\sigma_{1}}{\sigma_{1}^{2}+k^{2} t^{2}}-\kappa \frac{\sigma_{1}-1}{\left(\sigma_{1}-1\right)^{2}+k^{2} t^{2}} .
\end{aligned}
$$

Case I. If $k=0$, then $\Sigma_{k}$ is only dependent on $\sigma$, with a singularity occuring at $\sigma=1$. In fact,

$$
\Sigma_{0}(\sigma, t)=\frac{1}{\sigma}+\frac{1}{\sigma-1}-\frac{\kappa}{\sigma_{1}}-\frac{\kappa}{\sigma_{1}-1}:=\frac{1}{\sigma-1}+h(\sigma) .
$$

We observe that $h(\sigma)$ increases as $\sigma$ increases, so for $\alpha_{\varepsilon}=h(1+\varepsilon)<0.021467$, we have

$$
\Sigma_{0}(\sigma, t) \leq \frac{1}{\sigma-1}+\alpha_{\varepsilon} .
$$

Case II. Suppose $1 \leq k \leq 16$, then $\Sigma_{k}(\sigma, t)$ depends on $\sigma$ and $t$. For each $\sigma$, $\Sigma_{k}(\sigma, t)$ decreases as $t$ increases, because the derivative of $\Sigma_{k}(\sigma, t)$ with respect to $t$ is negative for all $t \geq 1$. Therefore, $\Sigma_{k}(\sigma, t) \leq \Sigma_{k}(\sigma, 1)$, which increases as $\sigma$ increases, because the derivative of $\Sigma_{k}(\sigma, 1)$ with respect to $\sigma$ is positive for all $1 \leq \sigma \leq 1.15$. It follows that

$$
\Sigma_{k}(\sigma, t) \leq \Sigma_{k}(1+\varepsilon, 1)<\mathcal{B}_{\varepsilon}(k),
$$

where admissible values for $\mathcal{B}_{\varepsilon}(k)$ are easily computed using a computer. To further verify this bound, the Maximize command in Maple confirms that the maximum of $\Sigma_{k}(\sigma, t)$ occurs at $\sigma=1+\varepsilon$ and $t=1$. For example, if $\varepsilon=0.15$ or $\varepsilon=0.01$, then admissible values of $\mathcal{B}_{0.15}(k)$ and $\mathcal{B}_{0.01}(k)$ are given in Table 1 and Table 2 respectively. Note that we round up at 8 decimal places, to account for any possible rounding errors.

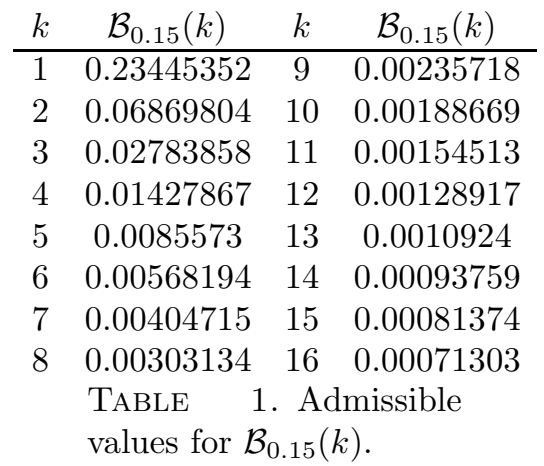

\begin{tabular}{cccc}
$k$ & $\mathcal{B}_{0.01}(k)$ & $k$ & $\mathcal{B}_{0.01}(k)$ \\
\hline 1 & 0.10919579 & 9 & 0.00029396 \\
2 & 0.03040152 & 10 & 0.00021655 \\
3 & 0.00958566 & 11 & 0.00016557 \\
4 & 0.00384196 & 12 & 0.00013046 \\
5 & 0.00185609 & 13 & 0.00010535 \\
6 & 0.00102853 & 14 & 0.00008684 \\
7 & 0.00063099 & 15 & 0.00007282 \\
8 & 0.00041809 & 16 & 0.00006196 \\
& TABLE & 2. Admissible \\
& values for $\mathcal{B}_{0.01}(k)$.
\end{tabular}

Now, we can collect the preceding observations to yield Lemma 5

Lemma 5. For $0 \leq k \leq 16$, we have that

$$
\Sigma_{k}(\sigma, t) \leq \begin{cases}\frac{1}{\sigma-1}+\alpha_{\varepsilon} & \text { if } k=0, \\ \mathcal{B}_{\varepsilon}(k) & \text { if } k \neq 0 .\end{cases}
$$


Under a choice of polynomial from $P_{16}$, it follows that

$$
S_{3} \leq a_{0}\left(\frac{1}{\sigma-1}+\alpha_{\varepsilon}\right)+\sum_{k=1}^{16} a_{k} \mathcal{B}_{\varepsilon}(k) .
$$

Remark. The benefits of Lemma[5]over [6, Lemma 2.4] lie in the computed constants $\mathcal{B}_{\varepsilon}(k)$. That is, Kadiri established $\Sigma_{k}(\sigma, t) \leq 1.6666$ for $1 \leq k \leq 4$.

2.3. Upper bound for $S_{4}$. We bring forward an observation from Kadiri [6, §2.4],

$$
\begin{aligned}
\mathfrak{R e}\left(\frac{\gamma_{L}^{\prime}\left(s_{k}\right)}{\gamma_{L}\left(s_{k}\right)}\right. & \left.-\kappa \frac{\gamma_{L}^{\prime}\left(s_{k}^{\prime}\right)}{\gamma_{L}\left(s_{k}^{\prime}\right)}\right) \leq-\frac{1-\kappa}{2} \cdot \log \pi \cdot n_{L} \\
& +\frac{n_{L}}{2} \max _{\delta \in\{0,1\}}\left\{\mathfrak{R e}\left(\frac{\Gamma^{\prime}}{\Gamma}\left(\frac{s_{k}+\delta}{2}\right)-\kappa \frac{\Gamma^{\prime}}{\Gamma}\left(\frac{s_{k}^{\prime}+\delta}{2}\right)\right)\right\} .
\end{aligned}
$$

Case I. If $k=0$, then we directly compute that

$$
\frac{1}{2} \max _{\delta \in\{0,1\}}\left\{\mathfrak{R e}\left(\frac{\Gamma^{\prime}}{\Gamma}\left(\frac{\sigma+\delta}{2}\right)-\kappa \frac{\Gamma^{\prime}}{\Gamma}\left(\frac{\sigma_{1}+\delta}{2}\right)\right)\right\} \leq d_{\varepsilon}(0),
$$

where $d_{\varepsilon}(0)$ is the maximum of the functions such that $\sigma=1+\varepsilon$. To see this, one can observe that the left-hand side of (9) is maximised at $\sigma=1+\varepsilon$ visually or use the Maximize command in Maple. For example, if $\varepsilon=0.01$, then

$$
d_{0.01}(0)=-0.2500763736 \text {. }
$$

Case II. Suppose $1 \leq k \leq 16$. McCurley [11, Lemma 2] establishes that

$$
\begin{aligned}
\frac{1}{2} & \mathfrak{R e}\left(\frac{\Gamma^{\prime}}{\Gamma}\left(\frac{s_{k}+\delta}{2}\right)-\kappa \frac{\Gamma^{\prime}}{\Gamma}\left(\frac{s_{k}^{\prime}+\delta}{2}\right)\right)=\frac{1-\kappa}{2} \log \frac{k t}{2}+\Xi(\sigma, k, t, \delta) \\
+ & \frac{\theta_{1}}{2 k}\left(\frac{\pi}{2}-\arctan \left(\frac{1+\delta}{k}\right)\right)+\kappa \frac{\theta_{2}}{2 k}\left(\frac{\pi}{2}-\arctan \left(\frac{\sigma_{1}(1)+\delta}{k}\right)\right),
\end{aligned}
$$

where $\left|\theta_{i}\right| \leq 1$ and

$$
\begin{aligned}
\Xi(\sigma, k, t, \delta)=\frac{1}{4} \log [ & \left.1+\left(\frac{\sigma+\delta}{k t}\right)^{2}\right]-\frac{\kappa}{4} \log \left[1+\left(\frac{\sigma_{1}+\delta}{k t}\right)^{2}\right] \\
& -\frac{\sigma+\delta}{2\left((\sigma+\delta)^{2}+k^{2} t^{2}\right)}+\kappa \frac{\sigma_{1}+\delta}{2\left(\left(\sigma_{1}+\delta\right)^{2}+k^{2} t^{2}\right)} .
\end{aligned}
$$

Next, we will bound $\Xi(\sigma, k, t, \delta)$ using two different methods, then choose the best bound for each $k$.

Method I. For any $t>0$, we have

$$
\begin{aligned}
\Xi_{1}(\sigma, k, t, \delta) & :=-\frac{\sigma+\delta}{2\left((\sigma+\delta)^{2}+k^{2} t^{2}\right)}+\kappa \frac{\sigma_{1}+\delta}{2\left(\left(\sigma_{1}+\delta\right)^{2}+k^{2} t^{2}\right)} \\
& \leq \frac{\kappa\left(\sigma_{1}+\delta\right)-\sigma-\delta}{2\left(\left(\sigma_{1}+\delta\right)^{2}+k^{2} t^{2}\right)} \leq 0
\end{aligned}
$$


because $\sigma<\sigma_{1}$ and $\kappa\left(\sigma_{1}+\delta\right)-\sigma-\delta \leq 0$. Moreover, for fixed $\sigma$, observe that

$$
\Xi_{2}(\sigma, k, t, \delta):=\frac{1}{4} \log \left[1+\left(\frac{\sigma+\delta}{k t}\right)^{2}\right]-\frac{\kappa}{4} \log \left[1+\left(\frac{\sigma_{1}+\delta}{k t}\right)^{2}\right]
$$

is positive for $t \geq 1$ and decreases as $t$ increases, because the derivative of $\Xi_{2}(\sigma, k, t, \delta)$ with respect to $t$ is negative for all $t \geq 1$. Therefore,

$$
\Xi_{2}(\sigma, k, t, \delta) \leq \Xi_{2}(\sigma, k, 1, \delta)
$$

for $t \geq 1$, which increases as $\sigma$ increases in the range $1 \leq \sigma \leq 1.15$, because the derivative of $\Xi_{2}(\sigma, k, 1, \delta)$ with respect to $\sigma$ is positive for $1 \leq \sigma \leq 1.15$. Hence, for each $k$,

$$
\Xi_{2}(\sigma, k, t, \delta) \leq \Xi_{2}(1+\varepsilon, k, 1, \delta) .
$$

To verify the preceding bound, the Maximize command in Maple confirms that the maximum of $\Xi_{2}(\sigma, k, t, \delta)$ occurs at $\sigma=1+\varepsilon$ and $t=1$. It follows that $\Xi(\sigma, k, t, \delta) \leq \Xi_{2}(1+\varepsilon, k, 1, \delta)$ for each $k$ and

$$
\frac{1}{2} \max _{\delta \in\{0,1\}}\left\{\mathfrak{R e}\left(\frac{\Gamma^{\prime}}{\Gamma}\left(\frac{s_{k}+\delta}{2}\right)-\kappa \frac{\Gamma^{\prime}}{\Gamma}\left(\frac{s_{k}^{\prime}+\delta}{2}\right)\right)\right\} \leq \frac{1-\kappa}{2} \log t+\mathcal{S}_{1}(k, \varepsilon),
$$

where $\mathcal{S}_{1}(k, \varepsilon)=\max _{\delta \in\{0,1\}}\left\{\mathcal{C}_{1}(k, \delta, \varepsilon)\right\}$ such that

$$
\begin{aligned}
\mathcal{C}_{1}(k, \delta, \varepsilon):= & \frac{1-\kappa}{2} \log \frac{k}{2}+\Xi_{2}(1+\varepsilon, k, 1, \delta) \\
& +\frac{1}{2 k}\left(\frac{\pi}{2}-\arctan \left(\frac{1+\delta}{k}\right)\right)+\frac{\kappa}{2 k}\left(\frac{\pi}{2}-\arctan \left(\frac{\sigma_{1}(1)+\delta}{k}\right)\right) .
\end{aligned}
$$

Method II. We will verify that for $0<\varepsilon \leq 0.15$,

$$
\Xi(\sigma, k, t, \delta) \leq \mathcal{A}(k, \delta, \varepsilon):= \begin{cases}0 & \text { if } \delta=0, \\ 0 & \text { if } \delta=1 \text { and } k \notin\{1,2\}, \\ \Xi(1+\varepsilon, k, 1,1) & \text { if } \delta=1 \text { and } k=1 . \\ \Xi(1.15, k, 1,1) & \text { if } \delta=1 \text { and } k=2 .\end{cases}
$$

First, for fixed $\sigma$ and $\delta=0$, the derivative of $\Xi(\sigma, k, t, \delta)$ with respect to $t$ is positive for $t \geq 1$, so $\Xi(\sigma, k, t, 0)$ is increasing as $t \rightarrow \infty$. Therefore, for each $\sigma \in[1,1.15]$,

$$
\Xi(\sigma, k, t, 0) \leq \lim _{t \rightarrow \infty} \Xi(\sigma, k, t, 0)=0 .
$$

Next, for fixed $\sigma$ and $\delta=1$, the derivative of $\Xi(\sigma, k, t, \delta)$ with respect to $t$ is positive for $t \geq 1$ whenever $k \notin\{1,2,3\}$, so $\Xi(\sigma, k, t, 1)$ is increasing as $t \rightarrow \infty$ for $k \notin\{1,2,3\}$. Therefore, for each $k \notin\{1,2,3\}$ and $1 \leq \sigma \leq 1.15$,

$$
\Xi(\sigma, k, t, 1) \leq \lim _{t \rightarrow \infty} \Xi(\sigma, k, t, 1)=0 .
$$

To completely verify (11), we now establish bounds for the special cases $\delta=1$ and $k \in\{1,2,3\}$. Observe that for each $t \geq 1$, the derivative of $\Xi(\sigma, k, t, 1)$ with respect to $\sigma$ is positive for $1 \leq \sigma \leq 1.15$ whenever $k \in\{1,2,3\}$, so

$$
\Xi(\sigma, k, t, 1) \leq \Xi(1+\varepsilon, k, t, 1) .
$$

Suppose that $k \in\{1,2,3\}$ and observe that in the range $t \geq 1, \Xi(1+\varepsilon, k, t, 1)$ either has one minimum point at $t=t_{k}(\varepsilon)$ or increases as $t \rightarrow \infty$. Here, $t_{k}(\varepsilon)$ equals the only root of the derivative of $\Xi(1+\varepsilon, k, t, 1)$ with respect to $t$ in the range $t \geq 1$. If this root does not exist, then set $t_{k}(\varepsilon)=1$ for convenience. For example, 
$t_{1}(0.15)=3.2308 \ldots, t_{2}(0.15)=1.6154 \ldots, t_{3}(0.15)=1.0769 \ldots$ and $t_{3}(0.01)=1$. It follows that $\Xi(1+\varepsilon, k, t, 1)$ decreases for $1 \leq t \leq t_{k}(\varepsilon)$ and $\Xi(1+\varepsilon, k, t, 1)$ increases for $t>t_{k}(\varepsilon)$, so

$$
\Xi(1+\varepsilon, k, t, 1) \leq \begin{cases}\Xi(1+\varepsilon, k, 1,1) & \text { if } 1 \leq t \leq t_{k}(\varepsilon), \\ \lim _{t \rightarrow \infty} \Xi(1+\varepsilon, k, t, 1) & \text { if } t>t_{k}(\varepsilon),\end{cases}
$$

in which $\lim _{t \rightarrow \infty} \Xi(1+\varepsilon, k, t, 1)=0$ for each $k$. If $k=1$, then for $t \geq 1$, we have

$$
\Xi(1+\varepsilon, 1, t, 1) \leq \max \{\Xi(1+\varepsilon, 1,1,1), 0\}=\Xi(1+\varepsilon, 1,1,1) .
$$

Observe that $\Xi(1+\varepsilon, 2,1,1)$ increases as $0<\varepsilon \leq 0.15$ increases. So, if $k=2$, then for $t \geq 1$, we have

$$
\begin{aligned}
\Xi(1+\varepsilon, 2, t, 1) \leq \max \{\Xi(1+\varepsilon, 2,1,1), 0\} & \leq \max \{\Xi(1.15,2,1,1), 0\} \\
& \leq \Xi(1.15,2,1,1) .
\end{aligned}
$$

In this case, the final bound is convenient and not too wasteful, because $\Xi(1.15,2,1,1)$ is small. Finally, if $k=3$, then for $t \geq 1$, we have

$$
\Xi(1+\varepsilon, 3, t, 1) \leq \max \{\Xi(1+\varepsilon, 3,1,1), 0\}=0 .
$$

Combining the observation (12) with (13), (14), and (15) implies (11). For each $k$, it follows from (10) and (11) that

$$
\frac{1}{2} \max _{\delta \in\{0,1\}}\left\{\mathfrak{R e}\left(\frac{\Gamma^{\prime}}{\Gamma}\left(\frac{s_{k}+\delta}{2}\right)-\kappa \frac{\Gamma^{\prime}}{\Gamma}\left(\frac{s_{k}^{\prime}+\delta}{2}\right)\right)\right\} \leq \frac{1-\kappa}{2} \log t+\mathcal{S}_{2}(k, \varepsilon),
$$

where $\mathcal{S}_{2}(k, \varepsilon)=\max _{\delta \in\{0,1\}}\left\{\mathcal{C}_{2}(k, \delta, \varepsilon)\right\}$ such that

$$
\begin{aligned}
\mathcal{C}_{2}(k, \delta, \varepsilon):= & \frac{1-\kappa}{2} \log \frac{k}{2}+\mathcal{A}(k, \delta, \varepsilon) \\
& +\frac{1}{2 k}\left(\frac{\pi}{2}-\arctan \left(\frac{1+\delta}{k}\right)\right)+\frac{\kappa}{2 k}\left(\frac{\pi}{2}-\arctan \left(\frac{\sigma_{1}(1)+\delta}{k}\right)\right) .
\end{aligned}
$$

Combination. We say that $\mathcal{S}(k, \varepsilon)=\min \left(\mathcal{S}_{1}(k, \varepsilon), \mathcal{S}_{2}(k, \varepsilon)\right.$ ) and (for $1 \leq k \leq 16$ ) present the quantities $\mathcal{S}_{1}(k, 0.15), \mathcal{S}_{2}(k, 0.15)$ and $\mathcal{S}(k, 0.15)$ alongside each other in Table 3 It turns out that $\mathcal{S}_{2}(k, 0.15)$ yields a better bound for cases $k=1,2,3,4$ and $\mathcal{S}_{1}(k, 0.15)$ yields the better bound otherwise. Finally, we package our observations into a useful lemma (Lemma 6).

Lemma 6. For $0 \leq k \leq 16$, we have shown that

$$
\mathfrak{R e}\left(\frac{\gamma_{L}^{\prime}\left(s_{k}\right)}{\gamma_{L}\left(s_{k}\right)}-\kappa \frac{\gamma_{L}^{\prime}\left(s_{k}^{\prime}\right)}{\gamma_{L}\left(s_{k}^{\prime}\right)}\right) \leq \begin{cases}n_{L}\left(d_{\varepsilon}(0)-\frac{1-\kappa}{2} \cdot \log \pi\right) & \text { if } k=0 \\ n_{L}\left(\frac{1-\kappa}{2}\left(\log t+\log \left(\frac{k}{\pi}\right)\right)+\mathcal{S}(k, \varepsilon)\right) & \text { if } k \neq 0\end{cases}
$$

Under a choice of polynomial from $P_{16}$, it follows that

$$
\begin{aligned}
S_{4} \leq a_{0} n_{L}\left(d_{\varepsilon}(0)-\right. & \left.\frac{1-\kappa}{2} \cdot \log \pi\right) \\
& +\sum_{k=1}^{16} a_{k} n_{L}\left(\frac{1-\kappa}{2}\left(\log t+\log \left(\frac{k}{\pi}\right)\right)+\mathcal{S}(k, \varepsilon)\right) .
\end{aligned}
$$




\begin{tabular}{c|c|c|c}
$k$ & $\mathcal{S}_{1}(k, 0.15)$ & $\mathcal{S}_{2}(k, 0.15)$ & $\mathcal{S}(k, 0.15)$ \\
\hline 1 & 0.3784516540 & 0.3249009026 & 0.3249009026 \\
2 & 0.3839873212 & 0.3763572015 & 0.3763572015 \\
3 & 0.4018562060 & 0.4004551145 & 0.4004551145 \\
4 & 0.4238223974 & 0.4236306767 & 0.4236306767 \\
5 & 0.4467597648 & 0.4468482525 & 0.4467597648 \\
6 & 0.4693610537 & 0.4695098183 & 0.4693610537 \\
7 & 0.4910902618 & 0.4912403488 & 0.4910902618 \\
8 & 0.5117562107 & 0.5118920810 & 0.5117562107 \\
9 & 0.5313238925 & 0.5314428586 & 0.5313238925 \\
10 & 0.5498280118 & 0.5499312088 & 0.5498280118 \\
11 & 0.5673323540 & 0.5674218683 & 0.5673323540 \\
12 & 0.5839104248 & 0.5839883668 & 0.5839104248 \\
13 & 0.5996362678 & 0.5997044990 & 0.5996362678 \\
14 & 0.6145802698 & 0.6146403531 & 0.6145802698 \\
15 & 0.6288074426 & 0.6288606647 & 0.6288074426 \\
16 & 0.6423769295 & 0.6424243440 & 0.6423769295
\end{tabular}

TABLE 3 . Computed values for $\mathcal{S}_{1}(k, 0.15), \mathcal{S}_{2}(k, 0.15)$ and $\mathcal{S}(k, 0.15)$.

Remark. The benefits of Lemma[6]over [6, Lemma 2.5] lie in the computed constants $d_{\varepsilon}(0)$ and $\mathcal{S}(k, \varepsilon)$. Kadiri imports results from McCurley [11, Lemma 2] for her bound, so the improvements we see follow from our observations pertaining to McCurley's work.

\begin{tabular}{l|l}
$a_{0}$ & 1 \\
$a_{1}$ & 1.74126664022806 \\
$a_{2}$ & 1.128282822804652 \\
$a_{3}$ & 0.5065272432186642 \\
$a_{4}$ & 0.1253566902628852 \\
$a_{5}$ & $2.372710620 \cdot 10^{-26}$ \\
$a_{6}$ & $2.818732841 \cdot 10^{-22}$ \\
$a_{7}$ & 0.01201214561729989 \\
$a_{8}$ & 0.006875849760911001 \\
$a_{9}$ & $2.064157910 \cdot 10^{-23}$ \\
$a_{10}$ & $6.601587090 \cdot 10^{-11}$ \\
$a_{11}$ & 0.001608306592372963 \\
$a_{12}$ & 0.001017994683287104 \\
$a_{13}$ & $6.728831293 \cdot 10^{-11}$ \\
$a_{14}$ & $3.682448595 \cdot 10^{-11}$ \\
$a_{15}$ & $2.949853019 \cdot 10^{-6}$ \\
$a_{16}$ & 0.00003713656497 \\
\hline
\end{tabular}

TABLE 4. Table of coefficients for Mossinghoff-Trudgian's polynomial $p_{16}(\varphi) \in P_{16}$.

2.4. Computations. As declared in the introduction, we will choose the polynomial $p_{16}(\varphi) \in P_{16}$ from [12, whose coefficients are given in Table 4, Suppose $r>0$ 
and $\sigma$ is chosen such that $\sigma-1=r(1-\beta)$ where $\rho=\beta+i t \in Z\left(\zeta_{L}\right)$ is an isolated zero such that $\beta \geq 1-\varepsilon \geq 0.85$. Applying the upper bounds for each $S_{i}$, which can be found in Lemmas 4, 5 and 6, then rearranging inequality (6) will yield

$$
\beta \leq 1-\frac{\frac{a_{1}}{1+r}-\frac{a_{0}}{r}}{c_{1} \log d_{L}+c_{2} n_{L} \log t+c_{3} n_{L}+c_{4}},
$$

where

$$
\begin{aligned}
& c_{1}=\frac{1-\kappa}{2} \sum_{k=0}^{16} a_{k}, \\
& c_{2}=\frac{1-\kappa}{2} \sum_{k=1}^{16} a_{k}, \\
& c_{3}=a_{0}\left(d_{\varepsilon}(0)-\frac{1-\kappa}{2} \log \pi\right)+\sum_{k=1}^{16} a_{k}\left(\frac{1-\kappa}{2} \log \left(\frac{k}{\pi}\right)+\mathcal{S}(k, \varepsilon)\right) \text { and } \\
& c_{4}=\alpha_{\varepsilon} a_{0}+\sum_{k=1}^{16} a_{k} \mathcal{B}_{\varepsilon}(k) .
\end{aligned}
$$

For the remainder of this proof, we replicate the process which Kadiri [6] followed. The maximum value of $\frac{a_{1}}{1+r}-\frac{a_{0}}{r}$ occurs at $r=\frac{\sqrt{a_{0}}}{\sqrt{a_{1}}-\sqrt{a_{0}}}$. Therefore, dividing the numerator and denominator of (16) by

$$
M=\frac{a_{1}}{1+\frac{\sqrt{a_{0}}}{\sqrt{a_{1}}-\sqrt{a_{0}}}}-\frac{a_{0}}{\frac{\sqrt{a_{0}}}{\sqrt{a_{1}}-\sqrt{a_{0}}}},
$$

we see that

$$
\beta \leq 1-\frac{1}{\frac{c_{1}}{M} \log d_{L}+\frac{c_{2}}{M} n_{L} \log t+\frac{c_{3}}{M} n_{L}+\frac{c_{4}}{M}} .
$$

In Table 5, we present the constants for two choices of $\varepsilon$. Observing the values for $\varepsilon=0.01$, inequality (17) will yield the explicit zero-free region (3) for $t \geq 1$, which completes the proof of Theorem 1

\begin{tabular}{l|ll} 
& $\varepsilon=0.15$ & $\varepsilon=0.01$ \\
\hline$M$ & 0.1021253857 & 0.1021253857 \\
$\frac{c_{1}}{M}$ & 12.24106100 & 12.24106100 \\
$\frac{c_{2}}{M}$ & 9.534650638 & 9.534650638 \\
$\frac{c_{3}}{M}$ & 0.444485082 & 0.050168175 \\
$\frac{c_{4}}{M}$ & 5.123026304 & 2.269182727
\end{tabular}

TABLE 5. Constants for the explicit zero-free region in Theorem 1 given $\varepsilon=0.15$ or $\varepsilon=0.01$. 


\section{Proof of Theorem 2}

Theorem 2 is an improvement of part of [6. Theorem 1.2]. We will recycle Kadiri's proof, except we use the polynomial $p_{16}(\varphi)$ in place of a polynomial from $P_{4}$. Suppose $\log d_{L}$ is asymptotically large and consider three regions,

$$
\mathbb{I}_{A}=\left(0, \frac{d_{1}}{\log d_{L}}\right], \mathbb{I}_{B}=\left(\frac{d_{1}}{\log d_{L}}, \frac{d_{2}}{\log d_{L}}\right], \mathbb{I}_{C}=\left(\frac{d_{2}}{\log d_{L}}, 1\right),
$$

where $d_{1}, d_{2}$ are constants to be chosen. Suppose further, that

$$
\sigma-1=\frac{r}{\log d_{L}} \text { and } 1-\beta=\frac{c}{\log d_{L}}
$$

In the regions $\mathbb{I}_{B}$ and $\mathbb{I}_{C}$, we impose further restrictions. Suppose $0<c, r<1$ such that

$$
\frac{a_{0}}{a_{1}-a_{0}} c<r \text { and } d_{2}>\frac{\sqrt{r(r+c)}}{2} .
$$

Combining analogous arguments to those results in [6, §3.2, §3.3, §3.4], one can easily establish that

in the region $\mathbb{I}_{A}$,

$$
0 \leq \frac{1}{r}-2 \frac{r+c}{(r+c)^{2}+d_{1}{ }^{2}}+\frac{1-\kappa}{2}
$$

$$
\begin{aligned}
0 \leq & \mathcal{E}_{B}\left(d_{1}, d_{2}, r, c\right) \\
& :=\frac{a_{0}}{r}-\frac{a_{1}}{r+c}+\frac{a_{1} r}{r^{2}+d_{1}{ }^{2}}-\frac{a_{0}(r+c)}{(r+c)^{2}+d_{1}{ }^{2}} \\
& -\frac{a_{0}(r+c)}{(r+c)^{2}+d_{2}{ }^{2}}-\frac{a_{1}(r+c)}{(r+c)^{2}+4 d_{2}{ }^{2}}+\frac{1-\kappa}{2} \sum_{k=0}^{16} a_{k} \\
& +\sum_{k=2}^{16} a_{k}\left(\frac{r}{r^{2}+k^{2}{d_{1}}^{2}}-\frac{r+c}{(r+c)^{2}+(k-1)^{2} d_{2}{ }^{2}}-\frac{r+c}{(r+c)^{2}+(k+1)^{2} d_{2}{ }^{2}}\right)
\end{aligned}
$$

in the region $\mathbb{I}_{B}$ and

$$
\begin{aligned}
0 & \leq \mathcal{E}_{C}\left(d_{2}, r, c\right) \\
& :=\frac{a_{0}}{r}-\frac{a_{1}}{r+c}+\frac{a_{1} r}{r^{2}+d_{2}{ }^{2}}-\frac{a_{0}(r+c)}{(r+c)^{2}+d_{2}{ }^{2}}+\frac{1-\kappa}{2} \sum_{k=0}^{16} a_{k}
\end{aligned}
$$

in the region $\mathbb{I}_{C}$. Suppose $d_{1}$ and $r$ are fixed. The admissible values of $c$ which one can input into (18) are those $c$ such that

$$
c \geq \frac{\sqrt{r^{2}-d_{1}^{2}\left(1+\frac{1-\kappa}{2} r\right)^{2}}-\frac{1-\kappa}{2} r^{2}}{1+\frac{1-\kappa}{2} r} .
$$

Denote the smallest value for $c$ in (21) by $c_{A}$. Next, let $c_{B}$ denote the root of $\mathcal{E}_{B}\left(d_{1}, d_{2}, r, c\right)$, where $r$ is chosen such that the root $c_{B}$ is as small as possible. Similarly, let $c_{C}$ denote the smallest root of $\mathcal{E}_{C}\left(d_{2}, r, c\right)$ for some optimally chosen $r$. It follows that $\zeta_{L}$ has at most one zero in the region $s=\sigma+i t$ such that $t<1$ and

$$
\sigma \geq 1-\frac{1}{R \log d_{L}}
$$


such that $R=\max \left(1 / c_{A}, 1 / c_{B}, 1 / c_{C}\right)$. Moreover, if an exceptional zero exists then it is real and simple by [6, §3.5]. To complete our proof of Theorem 2, it will suffice to show that $R=12.43436$ is an admissible value.

First, suppose that we choose the same values that Kadiri chose; $d_{1}=1.021$ and $d_{2}=2.374$. One can establish that $1 / c_{A}=12.5494$ when $r=2.1426$. Moreover, using our higher degree polynomial, we can compute the roots of $\mathcal{E}_{B}(1.021,2.374, r, c)$ and $\mathcal{E}_{C}(2.374, r, c)$ over a selection of $r$. The results of these computations are presented below.

\begin{tabular}{c|c|c} 
Root of & $r$ & $1 / c$ \\
\hline $\mathcal{E}_{B}(1.021,2.374, r, c)$ & 0.2366 & 12.43922 \\
$\mathcal{E}_{C}(2.374, r, c)$ & 0.2477 & 12.42548
\end{tabular}

Therefore, these choices of $d_{1}$ and $d_{2}$ would yield Theorem 2 with

$$
R=\max (12.5494,12.43922,12.42548)=12.5494 .
$$

Above, the limiting factor appears to be the value for $1 / c_{A}$. We can reduce the value of $1 / c_{A}$ by decreasing the value of $d_{1}$, however, we are also limited by the sizes of $1 / c_{B}$ and $1 / c_{C}$ which we can obtain. Therefore, we only need to choose $d_{1}$ such that $1 / c_{A}$ is small enough. The cost of choosing $d_{1}$ too small is a larger interval $\mathbb{I}_{B}$, which might not be ideal.

Given $d_{1}$, to find a good enough choice for $d_{2}$, we have tested many values for $d_{2}$ and computed the optimal outcomes in each case. If one chooses $d_{1}=1.0015$, then we found (to 3 decimal places) that $d_{2}=2.318$ yielded the best results. For this $d_{1}$, one can determine that $1 / c_{A}=9.7946$ when $r=2.1163$. The results of the remaining computations for $1 / c_{B}$ and $1 / c_{C}$ are presented below.

\begin{tabular}{c|c|c} 
Root of & $r$ & $\frac{1}{c}$ \\
\hline $\mathcal{E}_{B}(1.0015,2.318, r, c)$ & 0.2363 & 12.43355 \\
$\mathcal{E}_{C}(2.318, r, c)$ & 0.2473 & 12.43436
\end{tabular}

Therefore - as required - these choices of $d_{1}$ and $d_{2}$ will yield Theorem 2 with

$$
R=\max (9.7946,12.43355,12.43436)=12.43436 .
$$

\section{REFERENCES}

1. J.-H. Ahn and S.-H. Kwon, An explicit upper bound for the least prime ideal in the Chebotarev density theorem, Ann. Inst. Fourier 69 (2019), no. 3, 1411-1458.

2. N. Chebotarëv, Die Bestimmung der Dichtigkeit einer Menge von Primzahlen, welche zu einer gegebenen Substitutionsklasse gehören, Math. Ann. 95 (1926), no. 1, 191-228. MR 1512273

3. C. J. de la Vallée Poussin, Sur la fonction $\zeta(s)$ de Riemann et le nombre des nombres premiers inférieurs à une limite donnée. Mém. Acad. $r$, Mém. Couronnés et Autres Mém. Publ. Acad. Roy. Sci. Lett. Beaux-Arts Belg. 59 (1899), 74.

4. K. Ford, Zero-free regions for the Riemann zeta function, Number theory for the millennium, II (Urbana, IL, 2000), A K Peters, Natick, MA, 2002, pp. 25-56. MR 1956243 
5. D. R. Heath-Brown, Zero-free regions for Dirichlet L-functions, and the least prime in an arithmetic progression, Proc. London Math. Soc. (3) 64 (1992), no. 2, 265-338. MR 1143227

6. H. Kadiri, Explicit zero-free regions for Dedekind zeta functions, Int. J. Number Theory 8 (2012), no. 1, 125-147. MR 2887886

7. H. Kadiri, N. Ng, and P.-J. Wong, The least prime ideal in the Chebotarev density theorem, Proc. Amer. Math. Soc. 147 (2019), no. 6, 2289-2303.

8. N. M. Korobov, Estimates of trigonometric sums and their applications, Uspehi Mat. Nauk 13 (1958), no. 4 (82), 185-192. MR 0106205

9. J. C. Lagarias and A. M. Odlyzko, Effective versions of the Chebotarev density theorem, Algebraic number fields: $L$-functions and Galois properties (Proc. Sympos., Univ. Durham, Durham, 1975), 1977, pp. 409-464. MR 0447191

10. Y. V. Linnik, On the least prime in an arithmetic progression. II. The Deuring-Heilbronn phenomenon, Rec. Math. [Mat. Sbornik] N.S. 15(57) (1944), 347-368. MR 0012112

11. K. S. McCurley, Explicit zero-free regions for Dirichlet L-functions, J. Number Theory 19 (1984), no. 1, 7-32.

12. M. J. Mossinghoff and T. S. Trudgian, Nonnegative trigonometric polynomials and a zero-free region for the Riemann zeta-function, J. Number Theory 157 (2015), 329-349. MR 3373245

13. H. M. Stark, Some effective cases of the Brauer-Siegel theorem, Invent. Math. 23 (1974), 135-152. MR 0342472

14. S. B. Stečkin, The zeros of the Riemann zeta-function, Mat. Zametki 8 (1970), 419-429. MR 0280448

15. I. M. Vinogradov, A new estimate of the function $\zeta(1+i t)$, Izv. Akad. Nauk SSSR. Ser. Mat. 22 (1958), 161-164. MR 0103861

16. A. Zaman, Bounding the least prime ideal in the Chebotarev density theorem, Funct. Approx. Comment. Math 57 (2017), no. 1, 115-142.

unsw Canberra, Northcott Drive, Campbell, Canberra, ACT 2612 Australia 